\title{
Erratum to: Enzyme-amplified SERS immunoassay with Ag-Au bimetallic SERS hot spots
}

Xuan-Hung Pham ${ }^{1}$, Eunil Hahm ${ }^{1}$, Tae Han Kim ${ }^{1}$, Hyung-Mo Kim ${ }^{1}$, Sang Hun Lee ${ }^{1}$, SangChul Lee ${ }^{2}$, Homan $\mathrm{Kang}^{3}$, Ho-Young Lee ${ }^{2}$, Dae Hong Jeong ${ }^{4}$, Hak Soo $\mathrm{Choi}^{3}(\square)$, and Bong-Hyun Jun ${ }^{1}(\square)$

${ }^{1}$ Department of Bioscience and Biotechnology, Konkuk University, Seoul 143-701, Republic of Korea

${ }^{2}$ Department of Urology, Seoul National University Bundang Hospital, Seongnam 13620, Republic of Korea

${ }^{3}$ Gordon Center for Medical Imaging, Department of Radiology, Massachusetts General Hospital and Harvard Medical School, Boston, MA 02114, USA

${ }^{4}$ Department of Chemistry Education, Seoul National University, Seoul 151-742, Republic of Korea

(c) Tsinghua University Press and Springer-Verlag GmbH Germany, part of Springer Nature 2020

\section{Erratum to}

Nano Research 2020, 13(12): 3338-3346

https://doi.org/10.1007/s12274-020-3014-3

One author name and corresponding affiliation of the original version of this article were unfortunately mislabeled.

\section{Instead of}

Sang Chul Lee ${ }^{2}$

${ }^{2}$ Department of Nuclear Medicine, Seoul National University Bundang Hospital, Seongnam 13620, Republic of Korea
It should read

SangChul Lee ${ }^{2}$

${ }^{2}$ Department of Urology, Seoul National University Bundang Hospital, Seongnam 13620, Republic of Korea

The online version of the original article can be found at https://doi.org/10.1007/s12274-020-3014-3 